\title{
Análisis de la inversión extranjera directa como mecanismo de reactivación del sector productivo, periodo 2013-2017
}

\section{Analysis of foreign direct investment as a mechanism to reactivate the productive sector, 2013-2017 period}

\section{Análise do investimento estrangeiro direto como mecanismo de reativação do setor produtivo, período 2013-2017}

\author{
Zoila Filomena Pesantez-Cedeño ${ }^{\mathrm{I}}$ \\ zpesantezc@ulvr.edu.ec \\ Karla Karina Romero-Véliz II \\ karlae.g1489@outlook.com \\ Melba Ivonne Valdivieso-Salvatierra ${ }^{\text {III }}$ \\ iivonne_v36@hotmail.com
}

Recibido: 20 de marzo de 2019 *Aceptado: 15 de mayo de 2019 * Publicado: 05 de julio de 2019

1. Docente Investigador de la Carrera de Economía de la Facultad de Ciencias Sociales y Derecho de la Universidad Laica Vicente Rocafuerte de Guayaquil, Guayaquil Ecuador.

‥ Estudiante Egresada de la Carrera de Economía de la Facultad de Ciencias Sociales y Derecho de la Universidad Laica Vicente Rocafuerte de Guayaquil, Guayaquil Ecuador.

III. Estudiante Egresada de la Carrera de Economía de la Facultad de Ciencias Sociales y Derecho de la Universidad Laica Vicente Rocafuerte de Guayaquil, Guayaquil Ecuador. 


\section{Resumen}

Para el 2030 la UNESCO propone promover el crecimiento económico sostenido, y el trabajo decente para todos, en este sentido se presenta la investigación que aborda el comportamiento de la Inversión Extranjera Directa para la reactivación del empleo en el periodo de estudio. La indagación fue bibliográfica, descriptiva, de enfoque cuantitativo y los métodos utilizados fueron: estadístico, analítico y sintético. Entre los resultados más importantes: se evidencia que la IED y los resultados de la variable reactivación de la economía por generación de empleo se manifiesta de acuerdo al comportamiento de las distintas ramas que se detallan: En primer lugar se ubica la industria manufacturera (alimentos procesados) debido al impulso de la matriz productiva, seguido de agricultura, silvicultura, caza y pesca pese a que en los periodos anteriores se encontraba en los últimos lugares haciendo énfasis que la actividad que mayormente sobresale es la pesca, quedando en tercer lugar comercio (tiendas, restaurantes) actividad que se desarrolló en las principales ciudades de la zona 8 y 9 siendo la principal motivación de los inversionistas el número de habitantes y la dolarización, en cuarto lugar: servicios prestados a las empresas (actividades profesionales, técnicas y administrativas) que ha descendido debido a la insuficiencia de trabajadores técnicos calificados, y en quinto lugar la explotación de minas y canteras que ha descendido por las normativas de protección al medio ambiente que desalientan la IED en este sector.

Palabras clave: IED; reactivación económica; empleo; sectores económicos; normativas.

\section{Abstract}

By 2030, UNESCO proposes to promote sustained economic growth ... and decent work for all, in this sense the research that addresses the behavior of Foreign Direct Investment for the reactivation of employment in the study period is presented. The investigation was bibliographic, descriptive, of quantitative approach and the methods used were: statistical, analytical and synthetic. Among the most important results: it is evident that the FDI and the results of the variable reactivation of the economy by generating employment is manifested according to the behavior of the different branches that are detailed: First, the manufacturing industry is located (processed foods ) due to the impulse of the productive matrix, followed by agriculture, forestry, hunting and fishing although in the previous periods it was in the last places emphasizing that the activity that mostly 
stands out is fishing, being in third place commerce (stores, restaurants) activity that was developed in the main cities of zone 8 and 9 , the main motivation of investors being the number of inhabitants and dollarization, in fourth place: services provided to companies (professional, technical and administrative activities) that has declined due to the insufficiency of skilled technical workers, and fifthly the exploitation of mines and quarries that has declined due to environmental protection regulations that discourage FDI in this sector.

Key words: IED; economic reactivation; job; Economic sectors; normative.

\section{Resumo}

Até 2030, a UNESCO se propõe a promover o crescimento econômico sustentado ... e o trabalho decente para todos, neste sentido, a pesquisa que trata do comportamento do Investimento Estrangeiro Direto para a reativação do emprego no período do estudo é apresentada. A investigação foi bibliográfica, descritiva, de abordagem quantitativa e os métodos utilizados foram: estatístico, analítico e sintético. Entre os resultados mais importantes: é evidente que o IDE e os resultados da reativação variável da economia pela geração de emprego se manifesta de acordo com o comportamento dos diferentes ramos que são detalhados: Primeiro, a indústria de transformação está localizada (alimentos processados ) devido ao impulso da matriz produtiva, seguida pela agricultura, silvicultura, caça e pesca, embora nos períodos anteriores tenha ficado nos últimos lugares enfatizando que a atividade que mais se destaca é a pesca, sendo em terceiro lugar o comércio , restaurantes) atividade desenvolvida nas principais cidades das zonas 8 e 9 , sendo a principal motivação dos investidores o número de habitantes e a dolarização, em quarto lugar: serviços prestados às empresas (atividades profissionais, técnicas e administrativas) que diminuiu devido à insuficiência de trabalhadores técnicos qualificados, e quinto exploração de minas e pedreiras que diminuiu devido a regulamentações de proteção ambiental que desestimulam o IDE nesse setor.

Palavras-chave: IED; reativação econômica; emprego setores econômicos; normativo. 


\section{Introducción}

La UNESCO a través de la Agenda 2030 sugiere a los países a escala mundial y en especial a los menos desarrollados propiciar la generación del empleo decente en el crecimiento económico de los pueblos. De allí la necesidad de priorizar que la IED sea de calidad, que respete los derechos laborales de los trabajadores y sostiene: que es necesario “...fomentar políticas que estimulen el espíritu empresarial y la creación de empleo es crucial para este fin, así como también las medidas eficaces para erradicar el trabajo forzoso, la esclavitud y el tráfico humano ...”. (PNUD, 2015)

La IED definida como el ingreso de capitales percibidos por una nación, es relevante para mejorar las economías de menores ingresos en materia de desarrollo. En este sentido la investigación: "Análisis de la Inversión Extranjera Directa como mecanismo de reactivación del sector productivo, periodo 2013-2017" pretende conocer el comportamiento de la Inversión Extranjera Directa (IED) como mecanismo de reactivación en el país durante el periodo 2013-2017, para lo cual se abordaron importantes objetivos: investigar fundamentos teóricos que abordan el estudio, elaborar un cuadro comparativo de las normativas existentes antes y después del socialismo del siglo XXI, realizar una matriz FODA de la IED en el Ecuador e indagar qué sectores han sido los más y menos beneficiados con el ingreso de la IED y los eventos que motivaron la presencia o ausencia de ésta.

La Constitución del Ecuador (2008) ratifica la importancia de la IED y sostiene en el Art. 284 que la política económica debe “... Impulsar el pleno empleo y valorar todas las formas de trabajo, con respeto a los derechos laborales..." (Asamblea Nacional, 2008, p.92) En tanto que en el Art. 339 sobre las normas existentes que impulsan o detienen la inversión manifiesta “... será complementaria a la nacional, estará sujeta a un estricto respeto del marco jurídico y de las regulaciones nacionales, a la aplicación de los derechos y se orientará según las necesidades y prioridades definidas en el Plan Nacional de Desarrollo" (Asamblea Nacional, 2008, p.157)

De allí se justifica la investigación porque aporta valiosa información sobre las personas naturales o jurídicas extranjeras que buscan colocar su capital (dinero) en el país para crear nuevas formas de negocio (empresa-industrias) y con ello coadyuvar al fortalecimiento y desarrollo de la 
producción nacional, propendiendo a su vez a la generación de nuevas fuentes de empleo que tanto demanda la economía nacional.

\footnotetext{
${ }^{1}$ El trabajo decente sintetiza las aspiraciones de las personas durante su vida laboral. Significa la oportunidad de acceder a un empleo productivo que genere un ingreso justo, la seguridad en el lugar de trabajo y la protección social para las familias, mejores perspectivas de desarrollo personal e integración social, libertad para que los individuos expresen sus opiniones, se organicen y participen en las decisiones que afectan sus vidas, y la igualdad de oportunidades y trato para todos, mujeres $y$ hombres. (OIT, s.f.)
}

\section{Desarrollo}

Diversos autores opinaron que la IED es la clave para el progreso de países menos desarrollados, ya que a través de ella se busca nuevos mercados, por lo que constituye un aliado directo para fortalecer el comercio nacional e internacional.

La Teoría de Desarrollo de la Globalización (IED) es un referente teórico importante en el tema de la inversión porque fomenta el libre comercio, promueve la innovación tecnológica, en tal sentido Vargas (2008) manifiesta que "La teoría de la globalización enfatiza las transacciones económicas y sus vínculos políticos y financieros realizados con la complicidad del desarrollo de la tecnología de la información y la comunicación, desde una perspectiva de los elementos culturales".

El proceso de globalización tiene efectos positivos para las economías, así lo indica Cohen (1995) (citado en Vargas, 2008):

La globalización es un término que da cuenta de una doble realidad: de un lado la internacionalización de bienes, servicios y factores de producción; de otro, el surgimiento de empresas industriales capaces de concebir su desarrollo en escala mundial y formular para ello estrategias globales de producción, comercialización y gestión. (p.122)

En tanto que otros autores rechazan y manifiestan que la globalización es un proceso disímil, ya que no permite explotar la ventaja comparativa que poseen los diferentes países, debido a que está orientada hacia la parte financiera, tal es el caso de Vilas (1997) (citado en Vargas, 2008) que sustenta que "La globalización es un proceso de desarrollo desigual en sus diferentes niveles o dimensiones. En su etapa actual se encuentra mucho más desarrollada en materia financiera que en 
materia de producción o de comercio". En tanto que Chua (1998) (citado en Vargas, 2008) considera que este modelo no es favorable para la clase pobre debido a que tiene las siguientes condiciones: El subdesarrollo económico; Divisiones étnicas severas; La presencia dominante de una minoría étnica; La presencia de una mayoría empobrecida que como en el caso de los indígenas claman ser los dueños verdaderos de la nación y que ya empiezan a reconstruir su identidad.

Así también (Aguirre R, 2000) citado en Vargas, 2008 sostiene que el concepto de globalización es ambiguo y sólo trata de expresar las evidencias empíricas... En todo caso este concepto de globalización debe revisarse desde sus fundamentos, los contenidos y como procesos que caracterizan al desarrollo del capitalismo mundial y sociedades contemporáneas La globalización ha permitido que las economías de cada país se encuentren más relacionadas entre sí, ya sea por la libre circulación de capitales o por nuevos avances tecnológicos. Las economías nacionales toman como referencia los procesos mundiales, se integran cada vez más a los mercados globales que a los nacionales; es decir que todo país en vías de desarrollo debe buscar la apertura comercial para así poder obtener mayores beneficios.

La globalización y la IED tienen una relación positiva puesto que implica un ahorro interno, transferencia de tecnología, lo que permite al país competir tanto en el mercado local como internacional, alianzas con otros países, desarrollo de sector financiero son algunos de los factores positivos.

En tanto que la teoría de Desarrollo de Dependencia (Reactivación económica) surge en América Latina en 1960, para explicar el desarrollo económico de la región, debido a las medidas aplicadas en dichos países; décadas atrás debido a la crisis económica mundial de los años 30 , donde las economías latinoamericanas optaron por la industrialización, sustituyendo las importaciones de productos industriales que provenían del extranjero por productos nacionales.

Mientras algunos autores consideran que la pobreza de los países del antes llamados "tercer mundo" y hoy llamados países con ingresos bajos por la CEPAL, se debe a las empresas multinacionales y a las medidas proteccionistas adoptadas por los gobiernos de dichas naciones, así lo indica Cardozo y Faletto (1969) citado en Vargas, 2008 que considera que: 
Las diferentes regiones y países tienen intercambios desiguales en un sistema que concentra los recursos tecnológicos, la manufactura, la educación y la riqueza, mientras que otras regiones y países periféricos solo son proveedores de mano de obra y materia prima barata. (p.114)

Según Blomström y Ente (1990) (citado en Spicker, Alvarez, \& Gordon, s.f.), la teoría de dependencia sostiene los siguientes postulados: El subdesarrollo está directamente ligado a la expansión de los países industrializados; El desarrollo y subdesarrollo son dos aspectos diferentes de un mismo proceso; El subdesarrollo no es ni una etapa en un proceso gradual hacia el desarrollo ni una precondición, sino una condición en sí misma; La dependencia no se limita a relaciones entre países, sino que también crea estructuras internas en las sociedades.

Esta teoría defiende a la industria nacional, considera que mientras mayor desarrollo tenga el mercado nacional, se obtendrán mayores beneficios, por tal motivo (Vargas, 2008) considera que esta teoría "centra el desarrollo en los mercados domésticos, el papel del sector industrial nacional, generación de demanda agregada mediante incrementos salariales que aumentan los niveles de vida"(p.114); además el antes mencionado autor afirma que "la presión de la escasez lleva a las organizaciones a diferenciarse, a buscar la mayor posición de competitividad para adquirir recursos o para innovar en el uso de recursos alternativos".(p.116)

La teoría de Dependencia y la reactivación económica se relacionan entre si debido a que ambas promueven la industria nacional, puesto que buscan el desarrollo del mercado local, ya sea generando incentivos para las pequeñas y medianas empresas, para que no solo compitan a nivel nacional sino internacional; de esta forma se obtienen mayores beneficios para la ciudadanía.

\section{Metodología}

Para el estudio: Análisis de la Inversión Extranjera Directa como mecanismo de reactivación del sector productivo, periodo 2013-2017 fue necesario la utilización de la investigación bibliográfica, documental y descriptiva. Fue de enfoque cuantitativo porque se utilizó el método estadístico, analítico y sintético 
Las técnicas a emplear se relacionan con las fichas nemotécnicas que son aquellas que sirven para anexar los aspectos más importantes del contenido de un libro, de una revista o de un artículo periodístico tales como: conceptos, definiciones y comentarios. (Túqueres, s.f.) Para el ordenamiento de los datos y el análisis respectivo, en la presentación de resultados fue necesario indagar en documentos de importantes autores sobre las variables en documentos de la CEPAL, Banco Central del Ecuador, INEC, Constitución de la República del Ecuador, Cámara de Comercio de Guayaquil, Superintendencia de Compañías Valores y Seguros.

\section{Resultados y Análisis}

Los resultados que se presentan a continuación han sido extraídos del Banco Central del Ecuador del documento Boletín digital No 66, es importante destacar que esta institución tiene como uno de sus principales objetivos mantener actualizada la información macroeconómica del país. El análisis fue enriquecido con la información indagada sobre comentarios y opiniones de especialistas en el tema y que constan en páginas digitales sean estas revistas especializadas o artículos periodísticos.

\section{IED por país de origen}

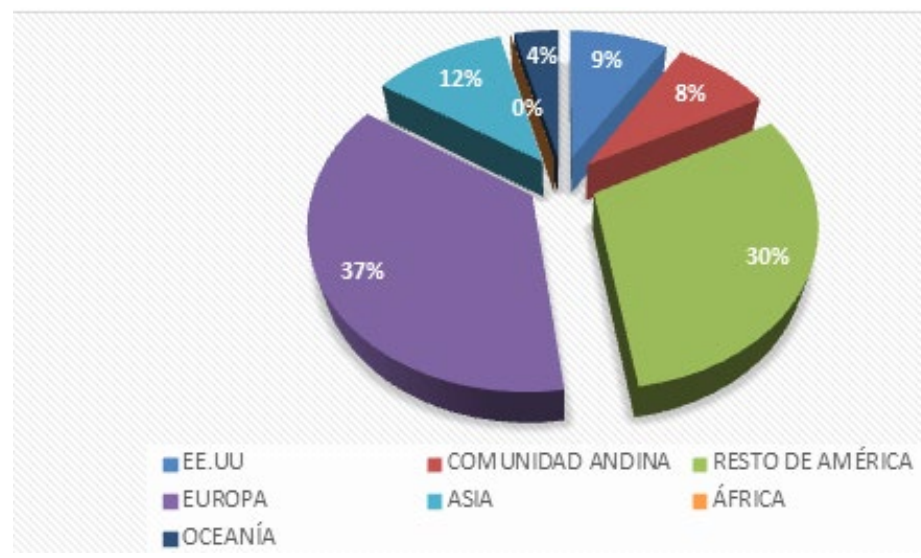

Figura 1. IED por país de origen

Fuente: Banco Central del Ecuador documento Boletín No 66 (2019) 


\section{Análisis}

La información proporcionada por la muestra evidenció que los países de Europa y el Resto de América fueron los invirtieron en mayor cantidad para el periodo analizado.

\section{La IED en la región 2016-2017}

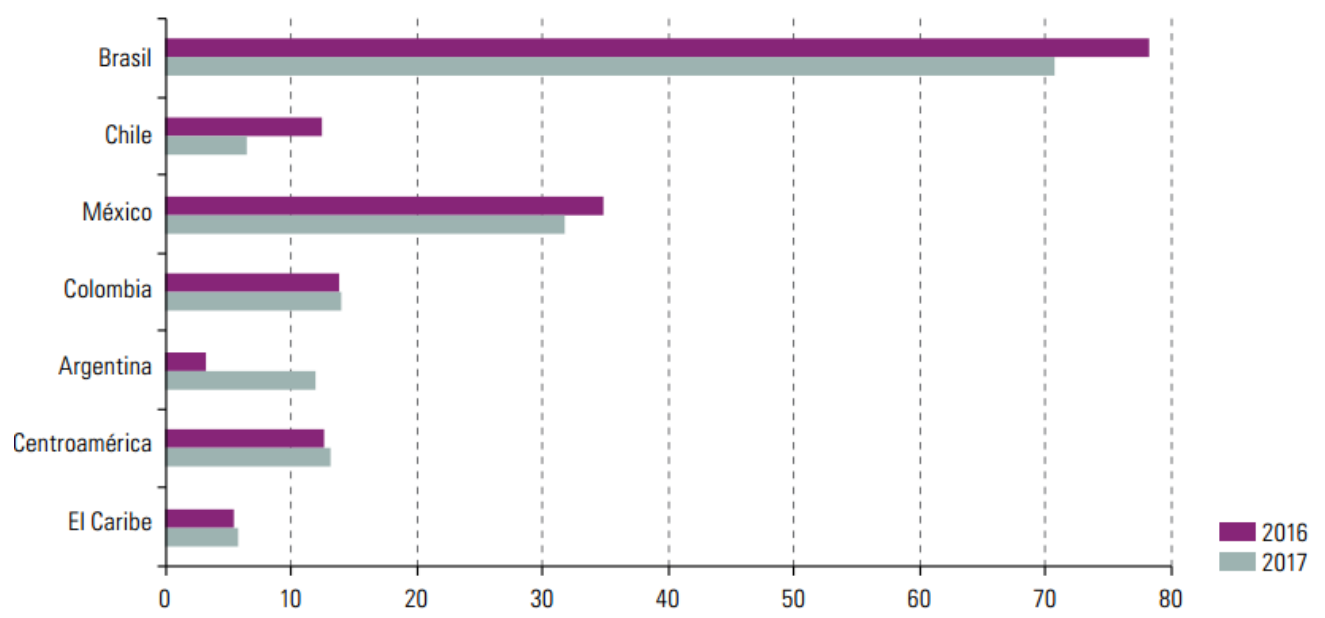

Figura 2. IED en la región 2016-2017

Fuente: Banco Central del Ecuador documento Boletín No 66 (2018)

\section{Análisis}

La IED en América Latina y el Caribe a nivel general se redujo en el año 2017, hasta los 161911 millones de dólares, esto se debe a la baja de los precios en los productos básicos de exportación, lo que provoco el descenso de la inversión en las industrias extractivas entre 2015 y 2016. (CEPAL, 2017)

\section{La IED del Ecuador por rama de actividad: Agricultura, silvicultura, caza y pesca}




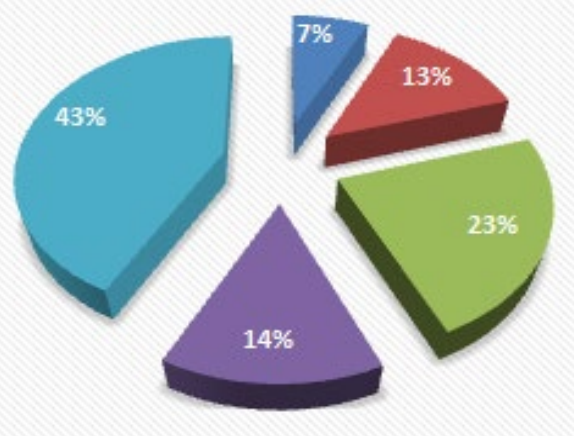

$$
\begin{array}{r}
=2013 \\
=2014 \\
=2015 \\
=2016 \\
=2017
\end{array}
$$

Figura 3. IED del Ecuador por rama de actividad: Agricultura, silvicultura, caza y pesca Fuente: Banco Central del Ecuador documento Boletín No 66 (2018)

\section{Análisis}

La rama de actividad económica: agricultura, silvicultura, caza y pesca al 2017 se encontró en segundo lugar, en el periodo de 2013 a 2015 los ingresos por IED aumentaron, en tanto que para el año 2016 sufrió una notable caída en menos de un cuarto del total invertido, para recuperarse en el 2017 de manera significativa; al comparar esta con otras ramas se puede observar que no ha logrado llamar la atención del inversor. Las provincias que sobresalieron en esta rama son: Manabí, Santo Domingo de los Tsáchilas, Santa Elena, Guayas, Los Ríos, Bolívar y Galápagos. En Cuanto a la reactivación económica por generación de empleo esta rama según el INEC (2017) reveló que pasó de 10,0\% en el año 2013 a 10,3\% en el año 2017, lo que significa que tuvo un ligero aumento; además cabe recalcar que se desconoce el número de personas que no cuentan con empleo formal. 


\section{La IED del Ecuador por rama de actividad: Comercio}

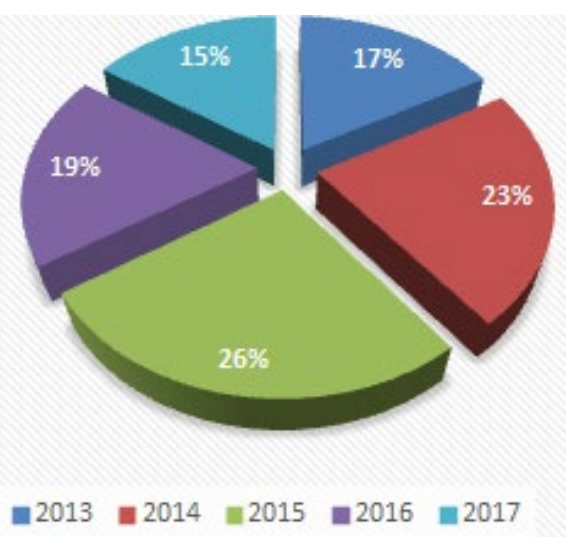

Figura 4. IED del Ecuador por rama de actividad: Comercio

Fuente: Banco Central del Ecuador documento Boletín No 66 (2018)

\section{Análisis}

La rama de actividad económica comercio que en 2017 ocupó el tercer lugar ha tenido un comportamiento irregular con subidas entre 2013 y 2015 aumentó en un monto mayor a un cuarto; siendo este último año en el que mayor captación de IED tuvo, entre 2016 y 2017 decreció hasta menos de un quinto debido a la abrupta caída del precio del petróleo en los mercados internacionales, situación que produjo inestabilidad en los puestos de trabajo, la importante inversión gubernamental se vio afectada y se contrajo la economía a nivel general. En lo que respecta al empleo adecuado según datos del INEC (2017) en 2013 tenía un 16,3\% y pasó a 17,5\% en 2017, cifra que reflejó un ligero aumento; esta rama fue la que mayor cantidad de participación de empleados generó. Las ciudades que se destacaron en la actividad comercial con mayor amplitud fueron: Guayaquil, Durán, Samborondón y Quito, debido al dinamismo comercial por ser Guayaquil en especial una ciudad portuaria. 


\section{La IED del Ecuador por rama de actividad: Construcción}

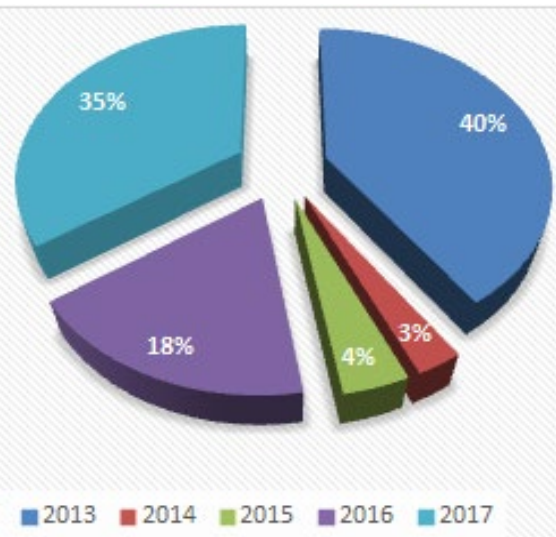

Figura 5. IED del Ecuador por rama de actividad: Construcción

Fuente: Banco Central del Ecuador documento Boletín No 66 (2018)

\section{Análisis}

La Construcción evidenció una fuerte contracción a partir del año 2014, pese a que en el 2013 obtuvo el mayor ingreso de IED del periodo de estudio, mayor al 40\% debido al auge que vivió el sector con las nuevas urbanizaciones cerradas y gracias al financiamiento oportuno del BIESS, en 2014 cayó significativamente la IED, producto de la inestabilidad económica que vivió el país a consecuencia de la caída abrupta del precio del petróleo. El sector fue recuperándose paulatinamente hasta llegar al 2017, sin que haya alcanzado la inversión recibida en el 2013. En lo que respecta al empleo adecuado según datos del INEC (2017) esta rama en 2013 generó un 9,7\% y paso a $8,1 \%$ al 2017, porcentaje que reflejó una disminución. Las ciudades en que se desarrolló esta rama con mayor auge fueron: Quito; Guayaquil y Samborondón respectivamente; siendo la primera la que tuvo mayor participación, debido a que representa la capital del Ecuador, sin menospreciar la importante inversión en la ciudad satélite. 


\section{La IED del Ecuador por rama de actividad: Electricidad, gas y agua}

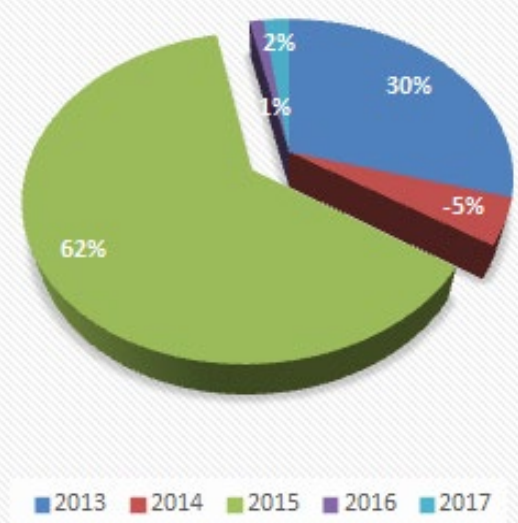

Figura 6. IED del Ecuador por rama de actividad: Electricidad, gas y agua

Fuente: Banco Central del Ecuador documento Boletín No 66 (2018)

\section{Análisis}

La rama de actividad económica: electricidad, gas y agua cerró el 2017 en octavo lugar y durante el periodo analizado presentó un comportamiento extremadamente atípico; éste guarda estrecha relación con la construcción de las hidroeléctricas edificadas en el país. Es así que el Bco. Central del Ecuador en sus estadísticas destacó a los años 2013 y 2015 como los de mayor IED, especialmente el año siguiente a la caída brusca del precio del petróleo. Las provincias que sobresalieron con las megas construcciones fueron: Pichincha, Carchi, Imbabura, Tungurahua, Cotopaxi y Cañar. En lo que respecta a la reactivación económica por generación de fuentes de trabajo fue de tipo público, y el empleo adecuado generado según el INEC (2017) paso de 1,2\% en 2013 a 1,3\% en 2017 porcentaje que representó un ligero incremento, sin dejar de resaltar el incremento de empleo en los dos años en que el gobierno recibió mayor IED a través de préstamos provenientes de China en su totalidad.

\section{La IED del Ecuador por rama de actividad: Explotación de minas y canteras}




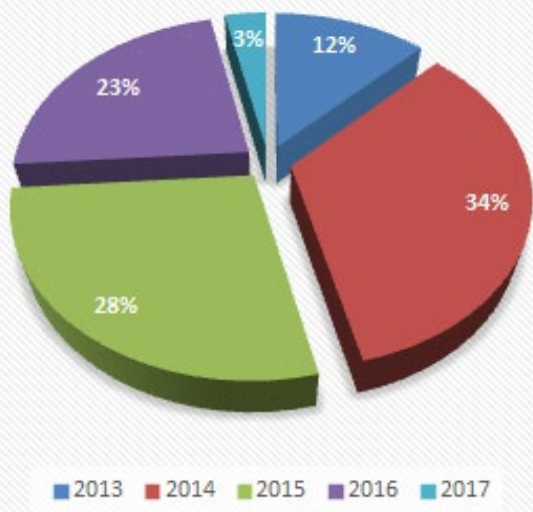

Figura 7. IED del Ecuador por rama de actividad: Explotación de minas y canteras

Fuente: Banco Central del Ecuador documento Boletín No 66 (2018)

\section{Análisis}

La rama: Explotación de minas y canteras fue una de las actividades económicas que mayor captación de IED obtuvo durante el periodo analizado, ya que del 2013 al 2014 casi se triplica la inversión, en tanto que debido a las nuevas normativas de protección al medio ambiente propuestas desde los Organismos internacional, hizo que la inversión decaiga a partir del 2014 al 2017; siendo su punto más alto el 2014 cuando obtuvo casi la mitad de lo invertido en el periodo de estudio en el Ecuador. Las provincias que se destacaron en esta rama fueron: El Oro, Pichincha, Azuay, Sucumbíos, Zamora Chinchipe, Guayas y Esmeraldas respectivamente. Esta rama se caracterizó por generar una captación importante y mayoritaria de empleo informal. En lo referente al empleo adecuado según datos del INEC (2017) para el año 2013 se registró un 1,2\% y un 0,7\% en 2017 este porcentaje es coherente con la disminución en la inversión que recibe la rama. 


\section{La IED del Ecuador por rama de actividad: Industria manufacturera}

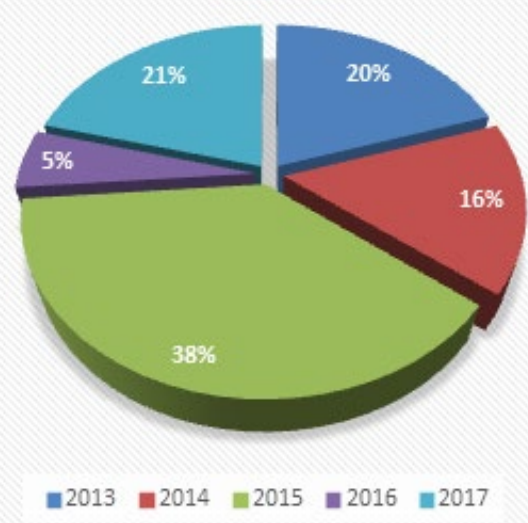

Figura 8. IED del Ecuador por rama de actividad: Industria manufacturera

Fuente: Banco Central del Ecuador documento Boletín No 66 (2018)

\section{Análisis}

La rama de actividad económica industria manufacturera en 2017 se encontró en primer lugar, durante el periodo analizado tuvo un comportamiento variado de 2013 a 2015; teniendo una ligera disminución en el 2014 para recuperarse en 2015; siendo este último el año en el que obtuvo mayor inversión por menos de la mitad del total invertido en esta rama; pese a la caída en 2016 en un 10\%, en 2017 aumentó cerca de un 20\%. La IED atraída por el sector manufacturero propició que la reactivación económica se vea reflejada por la generación de empleo adecuado en Ecuador según datos del INEC (2017) en 2013 tenía un 12,6\% paso a 13,0\% en 2017; aunque no tuvo un mayor incremento la industria manufacturera (elaboración de productos alimenticios, fabricación de caucho y plástico y elaboración de bebidas) es una de las ramas que generó mayor empleo. Las ciudades en las que esta rama se desarrolló mayormente son: Guayaquil y Quito; debido a que estas metrópolis cuentan con características únicas como Guayaquil considerada una ciudad portuaria.

\section{La IED del Ecuador por rama de actividad: Servicios comunales, sociales y personales}




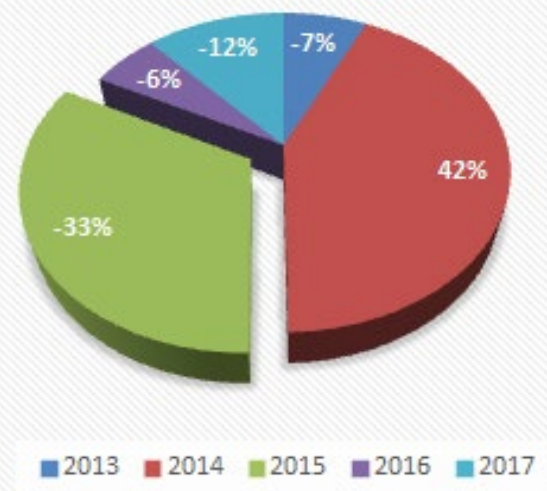

Figura 9. IED del Ecuador por rama de actividad: Servicios comunales, sociales y personales

Fuente: Banco Central del Ecuador documento Boletín No 66 (2018)

\section{Análisis}

La rama de actividad económica servicios comunales, sociales y personales en el año 2017 ocupó el noveno lugar, durante el periodo de estudio tuvo un comportamiento negativo de desinversión, excepto en el año 2014 que tuvo un porcentaje positivo. En lo que respecta al empleo adecuado según datos del INEC (2017) servicios comunales, sociales y personales en 2013 paso de 13,3\% a 12,9\% en 2017 cifra que disminuyó ligeramente, pese a ello esta rama estuvo entre las primeras en generar mayor empleo al Ecuador durante el periodo analizado. El Estado tuvo bajo control esta rama fue así que según la Secretaria Nacional de Gestión de la Política (2016) manifestó que se invirtió entre 2007-2016 treinta veces más que en gobiernos anteriores en educación a través de unidades educativas del milenio o por las becas otorgadas a estudiantes, salud en mejoras de infraestructura, servicios y defensa nacional mediante armamento y equipamiento. 


\section{La IED del Ecuador por rama de actividad: Servicios prestados a las empresas}

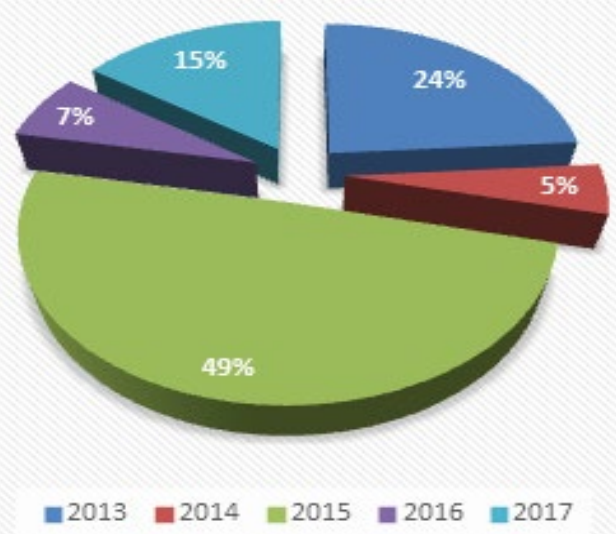

Figura 10. IED del Ecuador por rama de actividad: Servicios prestados a las empresas

Fuente: Banco Central del Ecuador documento Boletín No 66 (2018)

\section{Análisis}

La IED percibida por la rama de actividad económica servicios prestados a las empresas para el año 2017 ocupó el cuarto lugar; el comportamiento presentó fluctuaciones durante el periodo analizado de este modo de 2013 a 2017 aumentó; siendo el 2015 el año en el que mayor ingreso obtuvo con menos de la mitad y los años 2014 y 2016 tuvieron una disminución en menos de un décimo para recuperarse en 2017 con menos de un quinto. En lo que respecta al empleo adecuado según datos del INEC (2017) esta rama registró en 2013 un 6,8\% y 6,3\% en 2017, cifra que representó una ligera disminución, la misma ubicó a turismo entre las cinco ramas de actividad que genera mayor empleo; aunque se desconoce la cantidad de empleados que no poseen trabajo formal. Las provincias donde se desarrolló con mayor auge esta actividad fueron Manabí y Esmeraldas. 


\section{La IED del Ecuador por rama de actividad: Transporte, almacenamiento y comunicaciones}

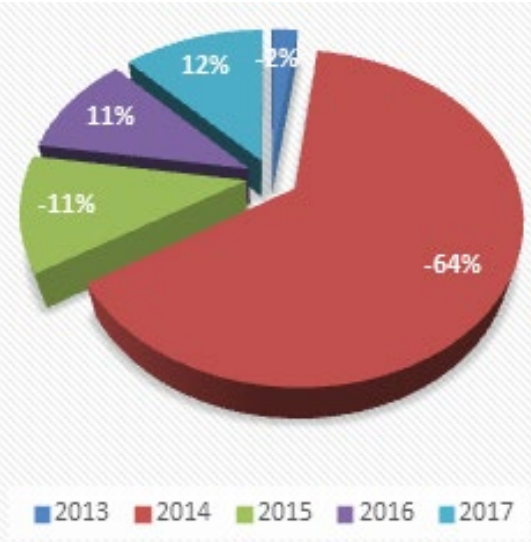

Figura 11. IED del Ecuador por rama de actividad: Transporte, almacenamiento y comunicaciones

Fuente: Banco Central del Ecuador documento Boletín No 66 (2018)

\section{Análisis}

La IED en transporte, almacenamiento y comunicaciones no presentó un ingreso significativo en comparación con otras ramas, al año 2017 ocupó el séptimo lugar; para el periodo de 2013 a 2015 tiene un porcentaje negativo, en tanto que entre 2015 y 2016 tuvo un ligero aumento de menos de un quinto. Ecuador debido a que sus costas se encuentran ubicadas sobre el Océano Pacifico generó beneficios para las empresas navieras en la transportación de sus cargas; además según Pro Ecuador (s.f.) cuenta con cuatro Autoridades Portuarias que fueron: Guayaquil, Manta, Esmeraldas y Bolívar; las mismas son manejadas por organismos estatales, siendo la de Guayaquil la que mayor cantidad de carga manejó. En lo que respecta al empleo adecuado según datos del INEC (2017) está rama durante el periodo de estudio pasó de 2013 que tenía un 7,1\% a 7,0\% en 2017, cifra que mostró que ha disminuido ligeramente. 


\section{Conclusiones}

La IED que Ecuador históricamente ha recibido es una de las más bajas del grupo de países que conforman la región, pese a ello, en el año 2015 se recupera significativamente con inversiones provenientes de EE.UU, Perú y China, debido a las estrategias que adoptó el gobierno de aquel entonces para minimizar el impacto de inestabilidad que vivía el país producto de los bajos precios internacionales del petróleo, para luego descender hasta el momento actual. Los países que han invertido de forma relevante son: Holanda, China, España y Uruguay. Las ramas de actividad económica que atraen inversión son: explotación de minas y canteras, industria manufacturera y comercio. Lo preocupante del tema es que las nuevas inversiones están buscando mercados más desarrollados donde haya los medios tecnológicos adecuados y el personal técnico altamente capacitado para producir bienes y servicios acorde a la era tecnológica vanguardista.

\section{Referencias Bibliográficas}

CEPAL. ( 2017). La Inversión Extranjera Directa en América Latina y el Caribe Recuperado el 30 de octubre de 2018, de Comisión Económica para América Latina y el Caribe: https://www.cepal.org/es/publicaciones/42023-la-inversion-extranjera-directa-america-latinacaribe-2017

Encalada, E. (2019). Las inversiones en turismo serán las más bajas en ocho años. El Comercio. Obtenido de https://www.elcomercio.com/tendencias/inversiones-turismo-ecuadorpresupuesto-ministerio.html

INEC. (2017). ENCUESTA NACIONAL DE EMPLEO, DESEMPLEO Y SUBEMPLEO. Obtenido de Intituto nacional de estadísticas y censos: http://www.ecuadorencifras.gob.ec/documentos/webinec/EMPLEO/2017/Diciembre/122017_M.Laboral.pdf

PNUD. (2015). Objetivo 8: Trabajo decente y crecimiento económico. Obtenido de Programa de las Naciones Unidas para el Desarrollo: 
https://www.undp.org/content/undp/es/home/sustainable-development-goals/goal-8-decentwork-and-economic-growth.html

PRO ECUADOR(s.f.). Negocios sin fronteras. Infraestructura Logistica. Obtenido de PRO ECUADOR: https://www.proecuador.gob.ec/infraestructura-logistica/

Secretaría Nacional de Gestión de la Politica. (2016). 70 mil millones de dólares invertidos en 9 años de Revolución. Obtenido de https://www.politica.gob.ec/70-mil-millones-de-dolaresinvertidos-en-9-anos-de-revolucion/

Spicker, P., Alvarez, S., \& Gordon, D. (s.f.). Teoría de la dependencia. Recuperado el 20 de diciembre de 2018, de Biblioteca virtual: http://bibliotecavirtual.clacso.org.ar/ar/libros/clacso/crop/glosario/t.pdf

Túqueres, S. (s.f.). Fichas nemotécnicas. Obtenido de Academia: https://www.academia.edu/9663468/Fichas_nemot\%C3\%A9cnicas

Vargas, J. (2008). Análisis crítico de las teorías del desarrollo. Recuperado el 22 de noviembre de 2018, de ResearchGate: https://www.researchgate.net/publication/23935088_ANALISIS_CRITICO_DE_LAS_TEORI AS_DEL_DESARROLLO_ECONOMICO 